\title{
3D Reconstruction of Coronary Artery using Biplane Angiography
}

\author{
Albert K. W. Law ${ }^{1}$, Hui $\mathrm{Zhu}^{2}$, and Francis H. Y. Chan ${ }^{1 *}$ \\ ${ }^{1}$ Biomedical Engineering Centre, Department of Electrical \& Electronic Engineering, \\ The University of Hong Kong, Hong Kong \\ ${ }^{2}$ Department of Biomedical Engineering, Duke University, NC, USA \\ *E-mail: fhychan@eee.hku.hk
}

\begin{abstract}
In this paper, we present a new method for the 3D reconstruction and visualization of coronary arteries in biplane angiography. The proposed method performs direct reconstruction of $3 \mathrm{D}$ coronary artery pathways without computing the 2D or 3D vessel centerlines. A front propagation algorithm is used to reconstruct the coronary artery pathways in 3D space. Starting from one or more 3D points, the front is expanded with a propagation speed controlled by the combined image information from two 2D projections. Then the reconstructed 3D coronary artery pathways are smoothed to reflect the real situation of a smoothed vessel surface. As shown in the experiment result, the coronary artery can be successfully reconstructed from two projections of biplane angiograms.
\end{abstract}

Keywords-3D reconstruction, biplane angiography, coronary artery, front propagation

\section{INTRODUCTION}

The visualization of $3 \mathrm{D}$ coronary artery pathways has attracted increasing attention in cardiac research in recent years. Quantitative coronary angiography (QCA) has been developed to apply computer technology for the $3 \mathrm{D}$ reconstruction and visualization of coronary arteries. It is useful for the diagnosis and assessment of coronary artery disease. The X-ray angiograms, which are projections of the $3 \mathrm{D}$ reality into a $2 \mathrm{D}$ representation, provide important information for the coronary artery reconstruction. The 3D pathways of coronary artery can be reconstructed from two or more $2 \mathrm{D} \mathrm{X}$-ray projection images.

Several methods have been developed for the 3D reconstruction and visualization of coronary arteries. Wahle et al. [1] initialized automated 2D vessel detection with manually placed bifurcation and vessel end points. The $3 \mathrm{D}$ information is derived using structural model correspondences. Chen and Carroll [2] used a modeling procedure for the reconstruction of $3 \mathrm{D}$ coronary arterial tree from at least two $2 \mathrm{D}$ projections. User interaction was needed to define the two dimensional centerlines and vessel widths. Haris et al. [3] proposed a method for the extraction and labeling of the coronary arterial tree using minimal user supervision in single view angiograms. Vessel overlap and foreshortening may significantly affect the extraction of $2 \mathrm{D}$ centerlines from individual projection images [4]. Young et al. [5] presented an approach for 3D vessel centerline direct extraction using a front propagation algorithm. A volume model of the coronaries can be constructed based on these axes and the $2 \mathrm{D}$ projection images.

In this paper, we present a new method for the $3 \mathrm{D}$ reconstruction and visualization of coronary arteries in biplane angiography. A 3D front propagation algorithm, guided by the combined image information from two $2 \mathrm{D}$ projections, is used to reconstruct the $3 \mathrm{D}$ coronary artery pathways. One or more points are selected on the vessel in one projection and their corresponding points in the other projection are found. The $3 \mathrm{D}$ positions of these points are reconstructed. Starting from these points, the front is expanded in $3 \mathrm{D}$ space with a propagation speed defined by combining the $2 \mathrm{D}$ vessel response of each projection. After that, the $3 \mathrm{D}$ coronary artery pathways are smoothed.

In section II, a detailed description of the new method is given. The experiment results are shown in section III. Finally, discussion and conclusion are presented in sections IV and $V$ respectively.

\section{METHODOLOGY}

\section{A. Overview}

Our method comprises of four main steps. The first step is image acquisition and preprocessing. Image acquisition is responsible for calibrating the biplane system and defining the projection transformations of the two projections. Preprocessing is contrast enhancement and pincushion distortion correction. The next step is to measure the $3 \mathrm{D}$ vessel response, which is defined by combining the image information of the two projections. Since the 2D image projections have an uneven background, an adaptive threshold, instead of a fixed threshold, is used for segmentation. Thus, the image information used in our method is the result from adaptive thresholding. The third step is front propagation for the reconstruction of the 3D coronary artery pathways. The final step is postprocessing for smoothing the vessel surface.

\section{B. Image Acquisition and Preprocessing}

Biplane coronary angiograms are acquired during routine clinical catheterization. A 3D Plexiglas cube, which contains twelve radio-opaque markers in known relation to one another, and a Plexiglas board embedded with a grid of radio-opaque balls are imaged at the same geometry as is used when capturing the artery images for the calibration of 
the biplane system and correction of the pincushion distortion of the images [6].

The preprocessing of the images includes: image contrast enhancement by linear gray level stretch; pincushion distortion correction using nonlinear transformations parameterized from the grid images; and calibration of the geometry of the imaging system.

A projection of a given $3 \mathrm{D}$ point onto a $2 \mathrm{D}$ point can be represented by

$$
\left(\begin{array}{l}
u^{i} \\
v^{i}
\end{array}\right)=\left(\begin{array}{l}
\frac{c_{11}^{i} x+c_{12}^{i} y+c_{13}^{i} z+c_{14}^{i}}{} \\
c_{31}^{i} x+c_{32}^{i} y+c_{33}^{i} z+c_{34}^{i} \\
\frac{c_{21}^{i} x+c_{22}^{i} y+c_{23}^{i} z+c_{24}^{i}}{c_{31}^{i} x+c_{32}^{i} y+c_{33}^{i} z+c_{34}^{i}}
\end{array}\right)
$$

where $(x, y, z)$ is the coordinate of the point in 3D space, and $\left(u^{i}, v^{i}\right)$ is its $2 \mathrm{D}$ coordinate in the projection plane $i$. The coefficients $c_{11}^{i}, c_{12}^{i}, \ldots, c_{34}^{i}$ can be solved when more than six markers of the calibration cube appear in the projected image. For the biplane angiography system, there are two projection transformations for the left and right anterior oblique (LAO and RAO) projections. The LAO projection is corresponding to the projection plane 1 while the RAO projection is corresponding to the projection plane 2 . The coefficients in these two transformations are found through the calibration cube images.

\section{A 3D Vessel Response Measure}

A vessel response measurement at a given $3 \mathrm{D}$ position can be defined by projecting the point into the $2 \mathrm{D}$ projection images, using the projection transformations of biplane angiography system as described above. In each projection, a $2 \mathrm{D}$ vessel response based on adaptive thresholding [7] is calculated. Adaptive thresholding can obtain the threshold surface, which is interpolated by using the gray level values at high gradient places of the original image. The $2 \mathrm{D}$ vessel response $R_{2 D}^{i}(u, v)$ may be defined as

$$
R_{2 D}^{i}(u, v)= \begin{cases}1, & \text { if } I^{i}(u, v)<T h^{i}(u, v) \\ 0, & \text { if } I^{i}(u, v) \geq T h^{i}(u, v)\end{cases}
$$

where $I^{i}(u, v)$ is the intensity value of $2 \mathrm{D}$ image and $T h^{i}(u, v)$ is the threshold surface at the point $(u, v)$ in the projection plane $i$.

The combined 3D vessel response $R_{3 D}(x, y, z)$ is defined according to the suitable characteristics of local image intensity distribution of the two $2 \mathrm{D}$ projections as

$$
R_{3 D}(x, y, z)=\prod_{i=1}^{n} R_{2 D}^{i}\left(u^{i}, v^{i}\right)
$$

where $R_{2 D}^{i}\left(u^{i}, v^{i}\right)$ is the $2 \mathrm{D}$ vessel response at the point $\left(u^{i}, v^{i}\right)$ in the projection plane $i$. The transformation relationship between the $3 \mathrm{D}$ point $(x, y, z)$ and the $2 \mathrm{D}$ point $\left(u^{i}, v^{i}\right)$ is described as in (1). For the biplane angiography system, $n$ is equal to two.

\section{Front Propagation}

A front propagation method [8] is used to extract the 3D coronary artery pathways. In this method, an interface is expanded through the volume of interest starting from a given initial boundary state (i.e. one or more points). The speed function of the propagation is controlled by the combined 3D vessel response $R_{3 D}(x, y, z)$.

The initial front is defined with one or more $3 \mathrm{D}$ points inside the vessel sections of interest. One or more points are selected on the vessel in one projection and their corresponding points on the vessel in the other projection are found under the constraint of the epipolar lines. The 3D positions of these points are reconstructed from their two projections and the caliblation parameters of the biplane imaging system.

Then the front propagation starts. The initial state of a time value $T(x, y, z)$ is defined as zero at the selected points, and infinity at all other unselected points. The border region is located between the selected and unselected regions. Their time values are updated according to the front propagation equation as follows:

$$
|\nabla T| R_{3 D}(x, y, z)=1
$$

where $R_{3 D}(x, y, z)$ is the combined 3D vessel response of the front and $T(x, y, z)$ is the time value when the front reaches the point $(x, y, z)$. The border with the lowest time value is moved into the selected region.

Stopping seeds are ser: at the end of the vessel sections of interest. The propagation stops as soon as the propagating front touches the stopping seeds.

\section{E. Postprocessing}

The 3D coronary artery pathways are extracted after front propagation. The vessel cross sections of the reconstructed coronary artery pathways are not smooth. This is because we only have two $2 \mathrm{D}$ projections. To reflect the real situation, the vessel is then smoothed by a 'box' filter with the size of the convolution kernel being [ [ $\left.\begin{array}{lll}3 & 3 & 3\end{array}\right]$. After the procedure, smoothed $3 \mathrm{D}$ coronary artery pathways are obtained.

\section{RESULTS}

In this section, we present the experiment result of our $3 \mathrm{D}$ reconstruction method applied to the angiograms of human coronary arteries. Fig. 1 shows the LAO and RAO projection pairs of a right coronary artery (RCA). Fig. 2 shows the result of adaptive thresholding described above applied to the LAO and RAO projections. The black region is the background with the $2 \mathrm{D}$ vessel response being zero. On the other hand, the white region is the vessel with the $2 \mathrm{D}$ vessel response being one. The main coronary artery was reconstructed by our method and the smoothed 3D coronary artery pathways at different view angles are shown in Fig. 3. 


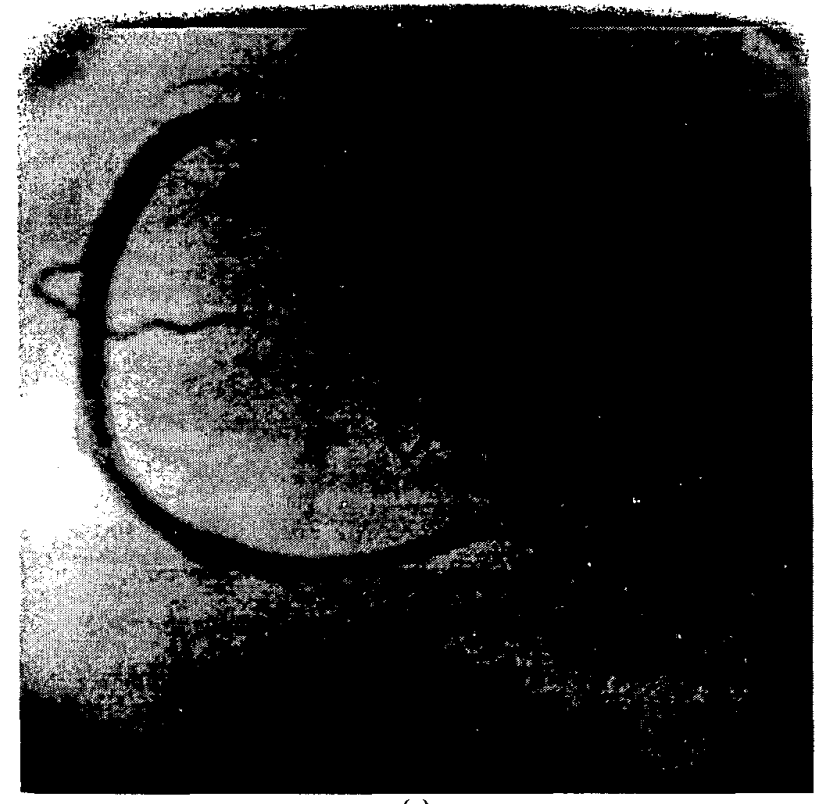

(a)

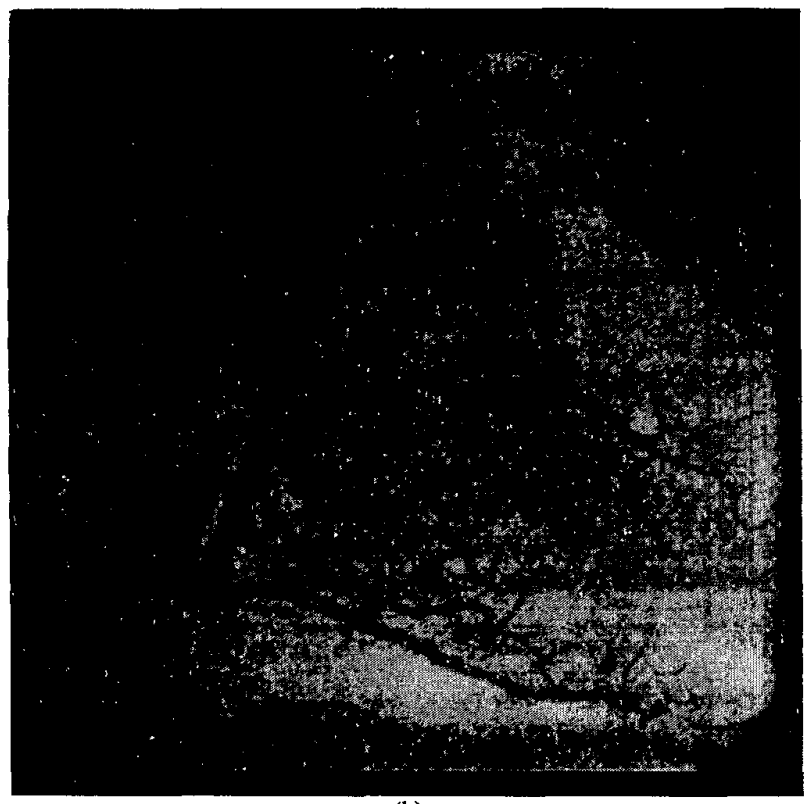

(b)

Fig. 1. The (a) LAO and (b) RAO projection pairs of a right coronary artery (RCA).

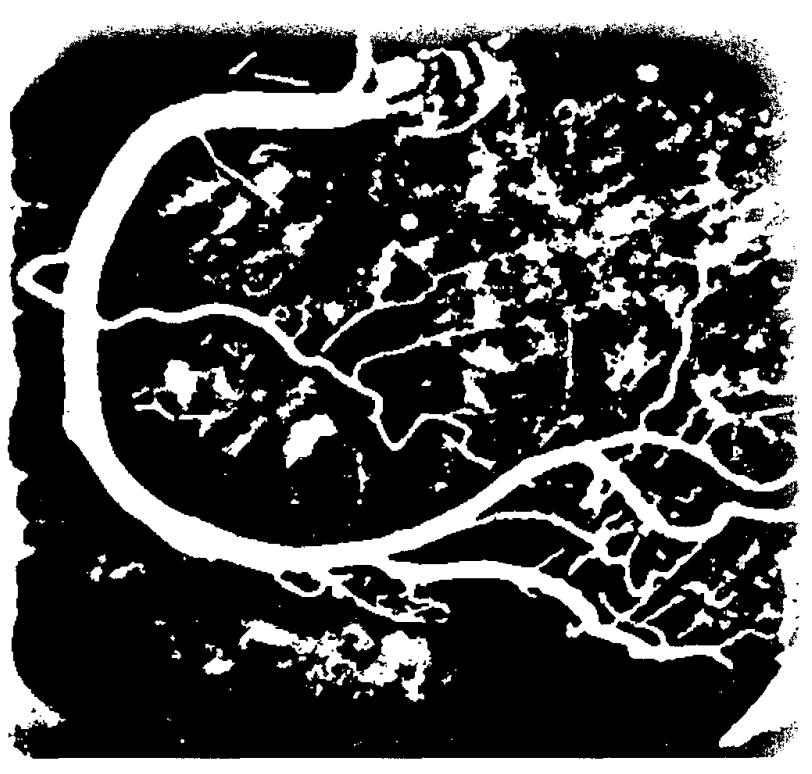

(a)

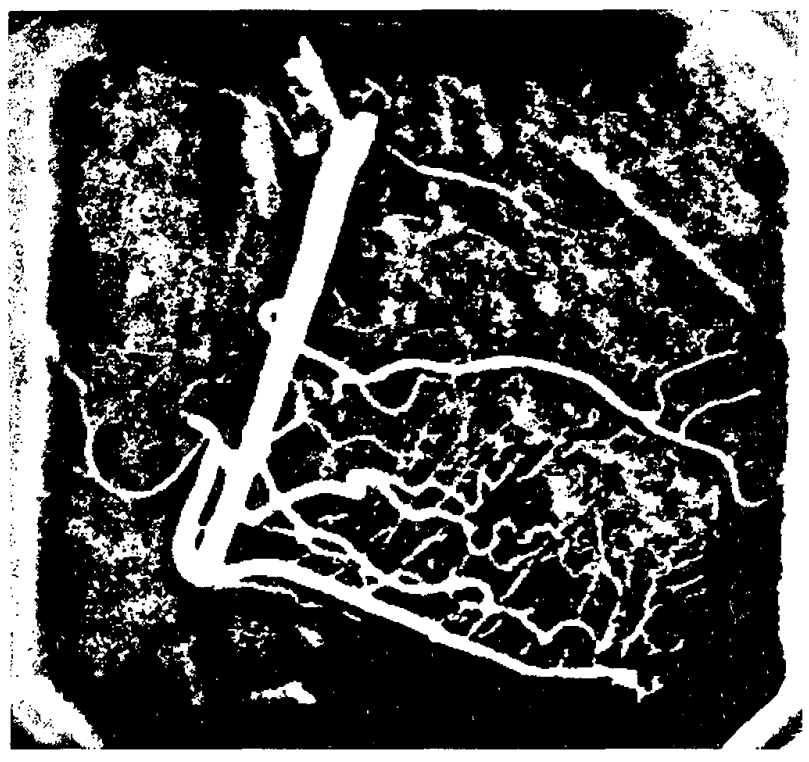

(b)

Fig. 2. The result of adaptive thresholding applied to the (a) LAO and (b) RAO projections. 

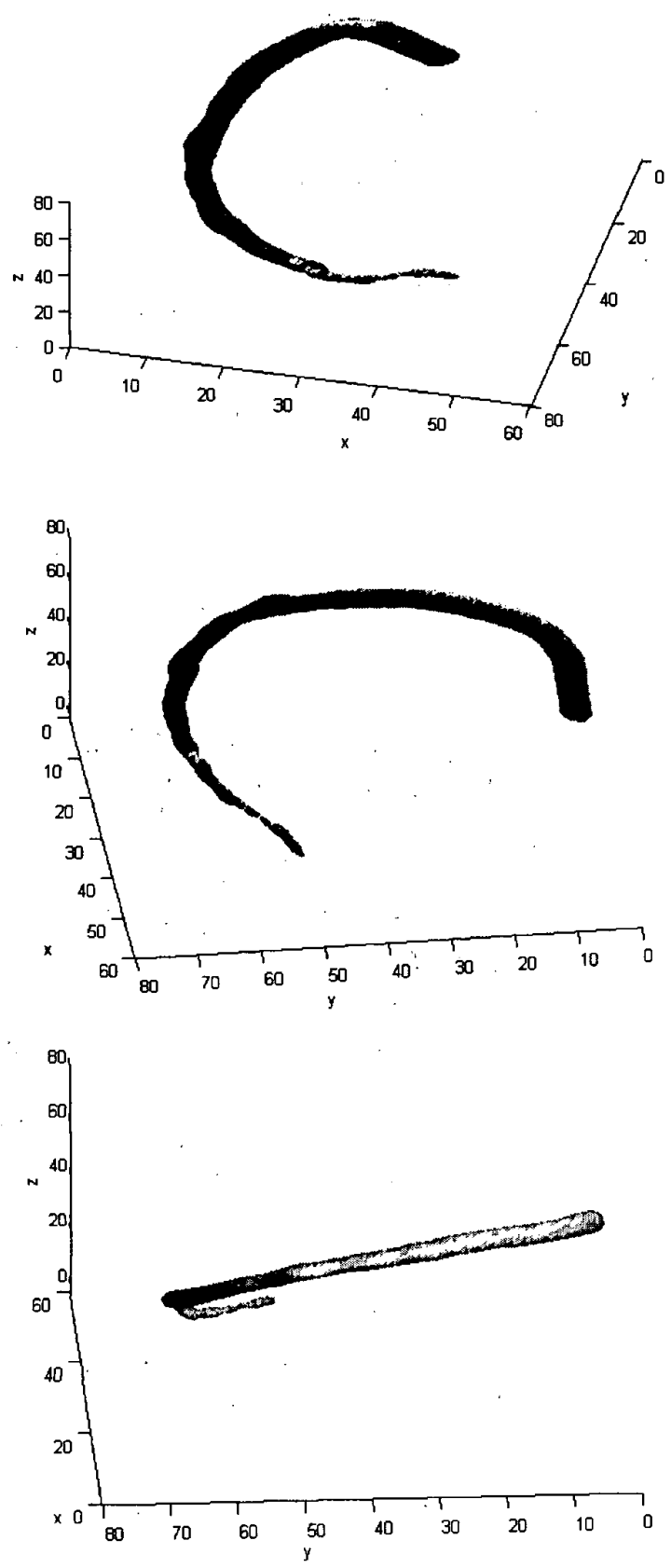

Fig. 3. The 3D coronary artery pathways at different view angles.

\section{Discussion}

There is an advantage of our 3D reconstruction method over those methods which reconstruct the vessel pathways from $2 \mathrm{D}$ or $3 \mathrm{D}$ centerlines. In our method, the $3 \mathrm{D}$ coronary artery pathways are extracted directly without the requirement of computing the $2 \mathrm{D}$ or $3 \mathrm{D}$ centerlines. The reconstructed 3D vessel pathways are then smoothed to reflect the real situation of a smoothed vessel surface.

The image information from two $2 \mathrm{D}$ projections is combined for the extraction of $3 \mathrm{D}$ vessel pathways. Problems of vessel overlap and foreshortening in individual projections are overcome by combining information from the two projections. Further improvement on the reconstruction results will be obtained if good quality projections, with less vessel overlap, can be used for the extraction of the $3 \mathrm{D}$ coronary artery pathways.

\section{ConClusion}

In this paper, we have presented a new method for the 3D reconstruction and visualization of coronary arteries in biplane angiography. More data sets are being tested on the performance of our method. It can be further developed for the quantitative analysis of coronary artery from biplane angiography.

\section{ACKNOWLEDGMENT}

We would like to thank Prof. Morton $\mathrm{H}$. Friedman at Duke University for providing the opportunity to work on this research project. Angiograms were acquired and provided by Dr. John J. Warner and Dr. Thomas R. Gehrig at Duke University Medical Center.

\section{REFERENCES}

[1] A. Wahle, E. Wellnhofer, I. Mugaragu, H. Sauer, H. Oswald, and E. Fleck, "Assessment of diffuse coronary artery disease by quantitative analysis of coronary morphology based upon 3D reconstruction from bijlane angiograms," IEEE Trans. Medical Imaging, vol. 14, pp. 230-241, Jun. 1995.

[2] S. J. Chen and J. D. Carroll, "3-D reconstruction of coronary arterial tree to optimize angiographic visualization," IEEE Trans. Medical Imaging, vol. 19, pp. 318-336, Apr. 2000

[3] K. Haris, S. N. Efstratiadis, N. Maglaveras, C. Pappas, J. Gourassas, G. Louridas, "Model-based morphological segmentation and labeling of coronary angiograms," IEEE Trans. Medical Imaging, vol. 18, pp. 1003-1015, Oct. 1999.

[4] H. Greenspan, M. Laifenfeld, S. Einav, and O. Barnea, "Evaluation of center-line extraction algorithms in quantitative coronary angiography," IEEE Trans. Medical Imaging, vol. 20, pp. 928-941, Sept. 2001.

[5] S. Young, B. Movassaghi, J. Weese, and V. Rasche, "3D vessel axis extraction using $2 \mathrm{D}$ calibrated $\mathrm{x}$-ray projections for coronary modeling," in Proc. SFIE Medical Imaging. San Diego, CA, Feb. 2003.

[6] H. Zhu and M. H. Friedman, "Tracking 3-D coronary artery motion with biplane angiography," in IEEE International Symposium on Biomedical Imaging, pp. 605-608, Washington, DC, Jul. 2002.

[7] F. H. Y. Chan, F. K. Lam, and H. Zhu, "Adaptive thresholding by variational method," IEEE Trans. Image Process., vol. 7, pp. 468-473, Mar. 1998.

[8] J. A. Sethian, Level Sat Methods and Fast Marching Methods: Evolving Interfaces in Computational Geometry. Fluid Mechanics, Computer Vision and Materials Science. Cambridge University Press, 1999 